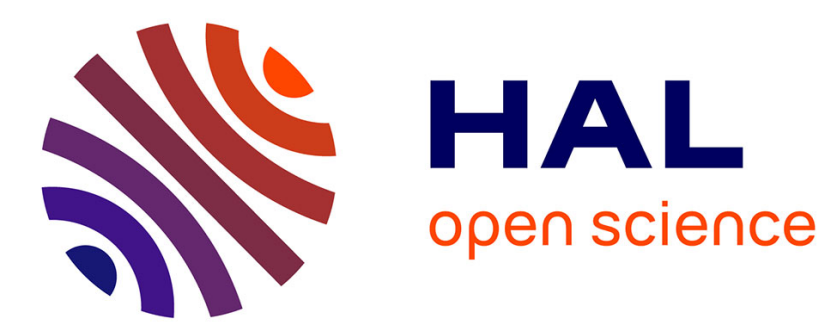

\title{
Des neurotechnologies duales?
}

Bernard Poulain

\section{To cite this version:}

Bernard Poulain. Des neurotechnologies duales?. 2021, 10.3917/rindu1.213.0053 . hal-03360654

\section{HAL Id: hal-03360654 \\ https://hal.science/hal-03360654}

Submitted on 30 Sep 2021

HAL is a multi-disciplinary open access archive for the deposit and dissemination of scientific research documents, whether they are published or not. The documents may come from teaching and research institutions in France or abroad, or from public or private research centers.
L'archive ouverte pluridisciplinaire HAL, est destinée au dépôt et à la diffusion de documents scientifiques de niveau recherche, publiés ou non, émanant des établissements d'enseignement et de recherche français ou étrangers, des laboratoires publics ou privés. 


\section{Bernard POULAIN}

Institut des Neurosciences Cellulaires et Intégratives

8 allée du général Rouvillois

67000 Strasbourg

Email : poulain@inci-cnrs.unistra.fr

\section{Des neurotechnologies duales ?}

Les neurotechnologies permettent d'enregistrer ou de modifier le fonctionnement du cerveau à des fins de recherche sur cet organe ou dans une perspective thérapeutique. D'autres applications des neurotechnologies visent à améliorer notre bien-être, jouer, éprouver des sensations fortes, améliorer nos apprentissages et nos performances... Les nouvelles applications de neurotechnologies surgissent si rapidement que nous ne prenons peut-être pas suffisamment le temps de nous interroger sur leur caractère éthique, sur leur utilité, sur les risques non justifiés qu'elles peuvent faire courir pour la santé de l'homme, sa sécurité, son autonomie et sa liberté. C'est la question du bon usage et du mesusage, de la dualité potentielle, des neurotechnologies qui est posée.

Ce texte vise à faire un bref rappel sur le fonctionnement électrique du cerveau et comment il est modifiable, notamment par des stimulations électriques. Les études d'efficacité clinique montrent que les neurotechnologies permettent d'obtenir des modifications durables du fonctionnement cognitif. Ceci met en question l'innocuité supposée de certaines neurotechnologies utilisées hors du domaine médical et nous questionne sur leur potentiel de mésusage.

Le fonctionnement "électrique " du cerveau est enregistrable et modifiable par des neurotechnologies.

Le transfert et le traitement des informations dans le cerveau sont basés sur des signaux " électriques " portés par des mouvements d'ions (atomes portant une charge électrique) au travers de la membrane plasmique des neurones. Ainsi les activités des neurones individuels, des réseaux qu'ils forment, des circuits et du cerveau global sont associées à des changements de potentiel électrique et du champ magnétique. L'exploration du fonctionnement et des dysfonctionnements du système nerveux à ses différentes échelles, du neurone au cerveau, est possible par diverses neurotechnologies (électrodes implantées, Electro EncéphaloGraphie (EEG), Magnéto-Encéphalo-Graphie (MEG)). Comme des transferts de charges électriques sont à la base du fonctionnement des cellules excitables, il est possible de modifier le fonctionnement du système nerveux par des stimulations électriques ou magnétiques pour activer les réseaux de neurones dans le cerveau (électrodes implantées, electrochocs; Stimulation Magnétique Transcrânienne -TMS) ou de moduler leur activité (stimulation électrique transcrânienne par courant continu (tDCS)). De nouvelles approches basées sur l'ingénierie génétique et moléculaire (opto-génétique etc) développée initialement à des fins de recherche en neurosciences permettent de concevoir de nouvelles interfaces utilisant des stimuli autres qu'électriques ou magnétiques. 
Le contrôle des mouvements et toutes les facultés de l'esprit humain telles que l'attention, la mémoire, la prise de décision, la navigation spatiale, la motivation, les émotions, la conscience de soi et d'autrui, les interactions sociales etc, c'est-à-dire tout ce qui forme la cognition et les comportements associés résultent de calculs produits dans divers circuits neuronaux $(1,2)$. Les neurotechnologies ne permettent pas d'agir sur la totalité des 100 milliards de neurones qui composent un cerveau humain. Leur but est de modifier certains aspects de son fonctionnement en stimulant ou modulant l'activité d'un circuit ou de quelques circuits et ainsi agir sur l'une ou plusieurs facultés cérébrales. Comme les changements d'activité des neurones et des circuits neuronaux induisent le plus souvent des mécanismes d'apprentissage de ces réseaux (par plasticité fonctionnelle voire anatomiques), l'effet des modulations ou des stimulations est souvent très durable dans le temps. En modifiant la cognition c'est la personne que l'on change ce qui n'est jamais une modification anodine. Seule des raisons médicales impératives permettent de le faire au seul bénéfice du patient.

\section{Dualités des applications médicales des neurotechnologies.}

Suite au procès de Nuremberg, un long travail de réflexion sur l'éthique a fait évoluer la loi (en France : Code civil et Code de la Santé Publique) encadrant la recherche sur la personne humaine, les applications et les indications médicales. Les dispositifs médicaux ou de recherche sur la personne humaine doivent être approuvés de façon préalable à leur utilisation. Leurs applications thérapeutiques doivent non seulement démontrer leur efficacité dans l'indication thérapeutique visée mais aussi démontrer que la balance bénéfice/risque est favorable au patient. Le cadre règlementaire est tout autant contraignant pour la recherche sur la personne humaine qu'elle soit interventionnelle ou non, c'est-à-dire invasive ou non. Quelle que soit la finalité de la recherche ou des applications thérapeutiques, il s'agit de répondre aux quatre grands principes de l'éthique -autonomie, bienfaisance, nonmalfaisance, justice- que nous rappelle Hervé Chneiweiss (3). Que ce soit dans un contexte de recherche ou d'intervention thérapeutique, la personne ou le patient doit être complètement informée des risques potentiels encourus afin de pouvoir donner un consentement éclairé à l'intervention qui est proposée.

Les neurotechnologies invasives comme la stimulation cérébrale profonde ou les implants cérébraux destinés à certaines interfaces cerveau machine (ICM) sont strictement réservées à des applications médicales (diagnostic, approches thérapeutiques en neurologie et psychiatrie). Le risque de mésusage concernera surtout les ICM invasives du futur car leur développement demandera au préalable d'avoir décodé le langage interne du cerveau (le code neural). En effet, coder l'information injectée vers le cerveau dans le " langage " naturel directement « intelligible » par le cerveau déchargera le patient implanté d'un engagement cognitif considérable. Ainsi, viendra le jour où devenus sourds et aveugles, grâce à nos prothèses sensorielles nous pourrons nous émerveiller à nouveau du concert donné par un pianiste virtuose tétraplégique équipé de prothèses des membres. Tout ceci est du domaine du rêve et il ne tiendra qu'à nous que ce rêve ne devienne pas un cauchemar. En effet, le décodage du code neural ouvrira la possibilité d'enfreindre la frontière ténue qui sépare la réparation, la réhabilitation des personnes, de l'augmentation, du transhumanisme voire de la prise de contrôle de la personne qui la transformerait en un cyborg déshumanisé. Le 
développement des ICM invasives et la recherche sur le décodage du code neural font clairement partie des domaines où la question de la dualité de la recherche est posée.

Demeurons optimistes mais vigilants : en ce qui concerne ces approches très invasives et très lourdes à mettre en œuvre mentionnées ci-dessus, le potentiel de détournement de leur usage vers un mésusage inacceptable est clairement identifié et ouvre de nouveaux défis éthiques, juridiques et sociaux (4) dont les principaux acteurs des neurotechnologies sont pleinement conscients (5).

\section{Dualité des applications non-médicales des neurotechnologies}

La neuromodulation -comme la tDCS- et le neurofeedback -une technologie ICM non-invasive, sont utilisées pour la recherche et dans certaines indications médicales. Leur caractère non invasif et leur bas coût a permis leur démocratisation et leur utilisation para-médicale ou grand public. Elles visent, par exemple, à améliorer le bien-être, l'intuition, la créativité, à faciliter ou améliorer les performances cognitives dans des domaines tels que l'éducation ou le jeu, à augmenter les performances des sportifs ou au travail. Ces utilisations échappent au cadre règlementaire strict appliqué à la recherche et à la clinique. Leur caractère non-invasif n'est pas synonyme d'innocuité. En effet, ces mêmes neurotechnologies ont été utilisées dans des indications thérapeutiques et leur évaluation selon les critères de l'evidence-basedmedicine a conclu à leur efficacité, au moins dans certaines indications. Par exemple la tDCS est efficace dans la dépression (niveau A) et probablement efficace (niveau B) dans certaines indications concernant la douleur, la maladie de Parkinson (motrice et cognitive), l'accident vasculaire cérébral (moteur), l'épilepsie, la schizophrénie et l'alcoolisme (6). Les mécanismes biologiques impliqués dans ces effets durables de la dTCS ne sont pas tous élucidés mais relèvent de la plasticité : remodelage du fonctionnement des réseaux de neurones et modification des taux d'expression de certaines protéines cérébrales (7-9). Pour le Neurofeedback, la qualité des études est souvent faible et ne permet pas d'établir complètement son efficacité $(10,11)$.

Qu'en est-il des formes de réalité immersives comme la réalité virtuelle, la réalité augmentée, ou encore la réalité étendue qui ont été conçues initialement pour des applications nonmédicales ? Les thérapies cognitives et comportementales (TCC) ont intégré avec succès les ressources ouvertes par la réalité virtuelle. La prise en charge des phobies (12-14) et de la dépression (15) avec approches incluant la réalité virtuelle est plus efficace que sans.

L'existence d'une efficacité thérapeutique de la neuromodulation, de la réalité virtuelle, voire du neurofeedback nous rassure parce que ceci valide l'utilisation de ces technologies dans les approches thérapeutiques de certaines indications. En même temps, cette efficacité nous alarme car il faudrait démontrer l'innocuité de ces approches, non seulement pour les patients dans la perspective de leur utilisation médicale, mais aussi pour les personnes saines en recherche et pour les utilisateurs du grand public. L'efficacité thérapeutique de la réalité virtuelle nous interpelle aussi sur le potentiel de mésusage des différentes formes de réalités immersives : est-ce que les multiples applications qui utilisent ces technologies peuvent être détournées pour modifier délibérément le comportement de leur utilisateurs (jeux video, formation, entraînement)? 


\section{Pour conclure,}

Les neurotechnologies et les nouvelles applications qu' elles offrent croissent très rapidement. Même si leur usage prévu est éthique, il nous faut anticiper les risques de leur détournement à des fins illégitimes (mésusage). II nous faut aussi évaluer le risque que leur utilisation en termes de conséquences inacceptables pour la santé et l'autonomie de l'humain. C'est la question de la dualité potentielle des neurotechnologies. Assez, paradoxalement, ce ne sont peut-être pas les applications les plus lourdes, les plus invasives qui sont les plus inquiétantes car le cadre règlementaire et les réflexions éthiques qui accompagnent leur développement ont identifié les limites à ne pas dépasser et les défis que leur développement a ouverts pour notre société. Les applications non-médicales et hors recherche académique des neurotechnologies, dans les domaines du jeu, de l'éducation, de la formation, etc, par manque d'évaluation préalable de leur innocuité, par méconnaissance de leur action potentiellement durable sur le cerveau, ou par mésusage délibéré, sont susceptibles de favoriser d'attenter à la vie privée cognitive (cognitive privacy) des personnes. II faudra donc identifier les mésusages potentiels des neurotechnologies afin d'y parer avant la diffusion de ces technologies et de leurs applications sur le marché.

\section{Références citées:}

1) Collins T, Andler D, Tallon-Baudry C, sous la direction de, 2018 La Cognition, Folio Essais.

2) Tallon-Baudry C, Cerveau et Cognition Chapitre 2, in Hirsch E, Poulain B., sous la direction de, 2019, Le Cerveau en Lumière, Odile Jacob.

3) Chneiweiss $\mathrm{H}$, La recherche et l'éthique, Chapitre 20, in Hirsch E , Poulain B., sous la direction de, 2019, Le Cerveau en Lumière, Odile Jacob.

4) https://www.oecd.org/fr/sti/tech-emergentes/recommandation-innovation-responsabledans-le-domaine-des-neurotechnologies.htm

5) http://brain.ieee.org/wpcontent/uploads/sites/52/2020/03/ieee brain neuroethics framework double-sided031920.pdf

6) Fregni F, El-Hagrassy MM, Pacheco-Barrios K, Carvalho S, Leite J, Simis V, Brunelin J, Nakamura-Palacios EM, Marangolo P, Venkatasubramanian G, San-Juan D, Caumo W, Bikson M, Brunoni AR, Neuromodulation Center Working Group. Evidence-Based Guidelines and Secondary Meta-Analysis for the Use of Transcranial Direct Current Stimulation in Neurological and Psychiatric Disorders. Int J Neuropsychopharmacol. 2021;24:256-313.

7) Nitsche MA, Paulus W. Sustained excitability elevations induced by transcranial DC motor cortex stimulation in humans. Neurology 2001. 57, 1899-1901.

8) Jamil A, Batsikadze G, Kuo H-I, Labruna L, Hasan A, Paulus W, Nitsche MA. Systematic evaluation of the impact of stimulation intensity on neuroplastic after-effects induced by transcranial direct current stimulation. J Physiol. 2017; 595:1273-1288.

9) Zhao X, Ding J, Pan H, Zhang S, Pan D, Yu H, Ye Z, Hua T. Anodal and cathodal tDCS modulate neural activity and selectively affect GABA and glutamate syntheses in the visual cortex of cats. J Physiol. 2020; 598:3727-3745. 
10) Jeunet C, Glize B, McGonigal A, Batail J-M, Micoulaud-Franchi J-A. Using EEG-based brain computer interface and neurofeedback targeting sensorimotor rhythms to improve motor skills: Theoretical background, applications and prospects. Neurophysiol Clin. 2019; 49:125136.

11) Patel K, Sutherland H, Henshaw J, Taylor JR, Brown CA, Casson AJ, Trujillo-Barreton NJ, Jones AKP, Sivan M. Effects of neurofeedback in the management of chronic pain: A systematic review and meta-analysis of clinical trials. Eur J Pain. 2020; 24:1440-1457.

12) Kahlon $S$, Lindner $P$, Nordgreen $T$. Virtual reality exposure therapy for adolescents with fear of public speaking: a non-randomized feasibility and pilot study. Child Adolesc Psychiatry Ment Health 2019 ; 13:47. 10.1186/s13034-019-0307-y

13) Lindner P, Miloff A, Bergman C, Andersson G, Hamilton W, Carlbring P. Gamified, Automated Virtual Reality Exposure Therapy for Fear of Spiders: A Single-Subject Trial Under Simulated Real-World Conditions. Front Psychiatry. 2020; 11:116. doi: 10.3389/fpsyt.2020.00116. eCollection 2020.

14) Kılıç A, Brown A, Aras I, Hui R, Hare J, Hughes LD, McCracken LM. Using Virtual Technology for Fear of Medical Procedures: A Systematic Review of the Effectiveness of Virtual Reality-Based Interventions. Ann Behav Med. 2021; Apr 3:kaab016. doi: 10.1093/abm/kaab016. Online ahead of print.

15) Fodor LA, Coteț CD, Cuijpers P, Szamoskozi Ș, David D, Cristea IA. The effectiveness of virtual reality based interventions for symptoms of anxiety and depression: A meta-analysis. Sci Rep. 2018; 8:10323. doi: 10.1038/s41598-018-28113-6. 